\title{
Measuring implicit alcohol associations via the Internet: Validation of Web-based implicit association tests
}

\author{
KatriJn Houben \\ Maastricht University, Maastricht, The Netherlands \\ AND \\ REINOUT W. WIERS \\ Maastricht University, Maastricht, The Netherlands \\ Radboud Universiteit, Nijmegen, The Netherlands \\ and IVO Addiction Research Institute Rotterdam, Rotterdam, The Netherlands
}

\begin{abstract}
Given the potential advantages of online assessment of implicit alcohol-related cognitive processes, the goal of this study was to empirically validate the online administration of the implicit association test (IAT). First, we examined whether an Internet-delivered IAT programmed in Flash can be as effectively used to assess implicit alcohol-related associations as equivalent IAT versions that are programmed in local lab software, such as Inquisit. Second, participants performed the IAT versions once in the controlled laboratory setting and once on their home computers via the Internet. Findings with the alcohol IAT versions were robust and did not vary systematically with respect to setting (home or lab) or assessment software (Flash or Inquisit). Importantly, there were also indications that IAT versions performed at home were more strongly related to explicit measures and drinking behavior than were lab-based IAT versions. Together, these findings demonstrate that the alcohol IAT can be validly administered online via participants' home computers.
\end{abstract}

Recently formulated dual-process models of addiction suggest that implicit or relatively automatic processes may play a central role in the etiology and maintenance of alcohol abuse (e.g., Deutsch \& Strack, 2006; Evans, 2003; Evans \& Coventry, 2006; Strack \& Deutsch, 2004; see Wiers \& Stacy, 2006a, for more examples). Specifically, the central notion of these models is that behavior is not only determined by explicit or deliberate processes, but also partly governed through relatively automatic processes that often exert their influence outside conscious control. Furthermore, it is proposed that addictive behaviors are determined by the dynamic interplay between two qualitatively different systems: a fast, associative impulsive system, which includes automatic appraisal of stimuli in terms of their affective and motivational significance, and a slower, rule-based reflective system, which includes controlled processes related to conscious deliberations, emotion regulation, and expected outcomes (e.g., Deutsch \& Strack, 2006; Strack \& Deutsch, 2004).

The development of these dual-process models of addictive behaviors has stimulated research on implicit processes that may play an essential role in alcohol abuse. This research has identified implicit (affective) memory associations as one of the implicit processes that may drive alcohol abuse. The idea is that once an alcohol-related cue is detected by an alcohol abuser, this automatically triggers implicit alcohol-related associations that are mostly affective in nature and that compel alcohol consumption. One of the most reliable methodologies for assessing such automatic or implicit memory associations is provided by the implicit association test (IAT; Greenwald, McGhee, \& Schwartz, 1998) and different varieties of this test (see Houben, Wiers, \& Roefs, 2006, for a review).

The IAT is a computerized classification task during which participants have to quickly and accurately classify stimuli into two target categories (e.g., alcohol and soft drinks) and two attribute categories (e.g., positive and negative), using a left and a right response key. Importantly, the target and attribute categories are assigned to the two response keys in two different combinations. For example, during the first combination task, participants have to press one response key for alcohol-related stimuli and positive stimuli and the other response key for softdrink-related stimuli and negative stimuli. This response assignment is then reversed in a second combination task, so that participants then have to respond to alcohol-related stimuli and negative stimuli with one response key and to soft-drink-related stimuli and positive stimuli with the other response key. The underlying idea is that this simultaneous classification of targets and attributes should be

K. Houben, k.houben@psychology.unimaas.nl 
easier when the response assignment of the target and attribute categories is compatible, or corresponds to respondents' implicit associations, than when this response assignment is incompatible, or does not match respondents' implicit associations. Hence, the difference in reaction time between the two combination tasks should reflect the strength of the associations of the target categories with the attribute categories. For example, faster performance when alcohol shares a response with positive attributes and soft drinks with negative attributes than when alcohol shares a response with negative attributes and soft drinks with positive attributes would indicate positive implicit associations with the target concept alcohol.

The common finding in studies in which the IAT has been used to study implicit alcohol-related associations has been that increased levels of alcohol consumption and alcohol abuse are related to stronger implicit associations between alcohol and positive affect, as well as to stronger implicit associations between alcohol and positive arousal (e.g., De Houwer, Crombez, Koster, \& De Beul, 2004; Houben \& Wiers, 2006, 2007a, 2007b; Wiers, van Woerden, Smulders, \& de Jong, 2002). Moreover, implicit alcohol associations with positive affect and arousal have been found to predict drinking behavior beyond the variance explained by explicit attitudes and expectations regarding the effects of alcohol (e.g., Houben \& Wiers, 2006, 2007a, 2007b; Wiers et al., 2002). Hence, these findings suggest that heavy drinkers have relatively strong implicit associations of positive affect and positive arousal that are activated automatically when they encounter alcoholrelated cues and that stimulate alcohol use and abuse. On the basis of these results and the ideas put forward in contemporary dual-process models, it is of clinical importance that existing interventions change not only explicit cognitions regarding drinking behavior, but also implicit associations. Moreover, on the basis of these new insights, it might also be possible to develop new interventions that are designed to target alcohol-related implicit processes (see Wiers et al., 2006, for some initial examples; for an overview, see Wiers et al., in press).

However, opportunities to further examine the role of implicit associations in alcohol abuse, as well as the effectiveness of both existing and new interventions in changing implicit alcohol-related associations, are compromised by several factors. First, research is typically confined to student populations, since it is generally easy to find students who are willing to participate in laboratory-based research, whereas nonstudent populations and clinical alcohol abusers are typically far more difficult to reach. Moreover, nonstudent and clinical populations are also often reluctant to take part in laboratory-based researchfor example, because they do not want to travel to the university where the research is conducted or because they are not convinced of the anonymity of participating in psychological research. Second, nonstudent populations and clinical populations may be more willing to participate in research outside of the laboratory (e.g., in the clinic where they are undergoing treatment). However, administering the IAT and related computerized measures of implicit processes outside of the laboratory typically presents technological difficulties. When conducting research that requires computerized assessment, the researcher has to have a computer at his disposal, which sometimes presents a problem outside of the laboratory. As a solution, the researcher can use a laptop (e.g., Ames et al., 2007; Thush et al., 2007), but even then, technical difficulties can hinder computerized assessment (e.g., problems with power supply). Finally, research on the effectiveness of interventions is often plagued by dropout, especially when the research design requires repeated sessions across an extended period of time.

Importantly, the development of the Internet and the Web now provides a new method of data collection that may overcome most of these problems of laboratorybased research. Online data collection is capable of reaching large numbers of nonstudent alcohol abusers, as well as clinical alcohol abusers, via their home computer and Internet connection, and in the complete anonymity and comfort of their own homes. Moreover, administering interventions that require repetition over an extended period of time over the Internet would no longer require participants to come to the lab on a daily basis and may, therefore, greatly diminish dropout. Finally, since context effects can have a profound influence on the assessment of alcoholrelated cognitive processes (e.g., Krank, Wall, Stewart, Wiers, \& Goldman, 2005), online measures of alcoholrelated cognitions in participants' home settings may be even more valid as compared with laboratory-based measurements, which are typically not alcohol related.

Despite these advantages, there are, of course, also downsides to administering implicit measures, such as the IAT, over the Internet. First, it needs to be established that the software used for online administration of implicit measures provides a measurement of reaction times that is as sensitive to differences in reaction times as software that is routinely used in the laboratory. Second, online testing makes it impossible to have a test setting that is standard for all participants. Specifically, testing via the Internet precludes the ability to set up the experiment in a standard test environment or via a standard computer setup. Moreover, with online testing, the experimenter no longer has control over participant behavior. Therefore, the goal of this study was to empirically validate the online administration of the IAT. Moreover, we wanted to verify the validity of both an IAT that measures implicit associations of alcohol with a bipolar affective attribute category (i.e., positive vs. negative; see, e.g., Houben \& Wiers, 2007b; Wiers et al., 2002) and an IAT that measures implicit associations of alcohol with positive arousal in a unipolar format (i.e., positive arousal vs. neutral; see, e.g., Houben \& Wiers, 2006).

First, we wanted to establish that the Internet-delivered IAT can be as effectively used to assess implicit alcoholrelated associations as an equivalent IAT that is programmed in standard software used in the lab (i.e., Inquisit). Specifically, the IAT measures implicit associations as the difference in reaction times between one combination of the target concepts with the attribute catego- 
ries (e.g., alcohol + positive vs. soft drinks + negative) and the reversed combination of the same categories (e.g., alcohol + negative vs. soft drinks + positive). It is, therefore, necessary to demonstrate that IAT effects obtained via the Internet do not differ from IAT effects measured using Inquisit. Furthermore, we also examined whether participants would commit similar numbers of errors in the online version of the IAT as in the Inquisit-based IAT. To examine the influence of software independently from the influence of setting, participants performed the bipolar IAT and the unipolar IAT either online in the laboratory or via Inquisit in the laboratory.

Second, we also examined the influence of testing implicit associations in the laboratory, as compared with testing implicit associations in a nonstandard setting that is no longer under the experimenter's control. Therefore, we examined whether online versions of the bipolar IAT and the unipolar IAT that are performed on one's home computer via the Internet would yield similar results, as compared with the lab-based IAT versions. Finally, we also wished to show comparable predictive validity for the IATs performed at home and the IATs performed in the laboratory. Predictive validity of IAT versions that are performed at home via the Internet may differ from the predictive validity of lab-based IAT versions, due to the uncontrollability of the home test environment and/or context effects that operate in the home environment but not in the standard lab setting. Therefore, the present study also examined whether implicit associations measured in the setting of participants' own homes would yield a relationship to drinking behavior similar to that of implicit associations that are measured within a standard laboratory setting.

\section{METHOD}

\section{Participants}

Participants were recruited through posters and flyers asking for experimental volunteers, distributed in and near the buildings of Maastricht University, and through advertisements posted in local newspapers. The participants were included only if they indicated that Dutch was their first language and were excluded if they indicated that they were color-blind or dyslexic. This resulted in a final sample that consisted of 115 participants. The mean age of the sample was 25.52 years $(S D=11.74)$. Average alcohol consumption per week was $6.91(S D=8.66)$ Dutch standard drinks. ${ }^{1}$ On the Alcohol Use Disorder Identification Test (AUDIT; Saunders, Aasland, Babor, De la Fuente, \& Grant, 1993), participants' mean score was $6.18(S D=4.03)$. An often-used cutoff score for the screening of alcohol-related problems is 11 (Saunders et al., 1993).

\section{Materials and Measures}

Implicit Association Test. During each session, the participants performed both a bipolar IAT version and a unipolar IAT version. In both IAT versions, two target categories were presented, one related to alcohol and the other to soft drinks. The alcohol target category consisted of six alcoholic drinks (wine, beer, pint, vodka, breezer [i.e., mixed drink], and whisky; label, alcohol), while the soft drink target category consisted of six soft drinks (Coca Cola, Fanta, orange soda, cassis, juice, and water; label, soft drinks). The two (Dutch) target categories were matched on number of syllables. Furthermore, in the bipolar IAT, a positive attribute category consisting of six general positive words (love, sunshine, warmth, peace, hug, and rainbow; label, pleasant) was paired with a negative attribute category that consisted of six general negative words (sorrow, war, depression, pain, fight, and disease; label, unpleasant). The (Dutch) positive and negative attribute categories were matched on number of syllables. In the unipolar IAT, one attribute category consisted of six words related to the arousing effects of alcohol (talkative, excited, cheerful, happy, funny, and energetic; label, active), which was paired with a neutral attribute category (average, indefinite, general, normal, habitual, and ordinary; label, neutral). The (Dutch) arousal category and neutral category were matched on number of syllables.

Both the bipolar IAT and the unipolar IAT followed the standard IAT procedure (see Table 1; cf. Greenwald et al., 1998). In the first block, the participants practiced the target discrimination. During this block, the participants classified target stimuli into the alcohol category or the soft drink category, using a left and a right response key. All the target stimuli were presented twice, resulting in 24 trials. In the second block, participants received 24 trials of attribute discrimination practice. In this block, the participants classified all the attribute stimuli as positive or negative, using the left and right response keys. All the attribute stimuli were presented twice. The third block consisted of 24 trials during which the participants practiced the combined classification of the target and attribute categories. During this combination practice, both target and attribute stimuli were presented. The participants had to classify stimuli belonging to one target category and one attribute category (e.g., alcohol and positive) with one response key. Stimuli belonging to the other target category and the other attribute category (e.g., soft drinks and negative) had to be classified by pressing the other response key. All the target and attribute stimuli were presented once. The fourth block was the combination test block, for which the instruction was the same as in the third block. During this block, all the target and attribute stimuli were presented twice, for a total of 48 trials. During the fifth block, the response assignment of the target categories was reversed. The participants practiced this reversed target discrimination during 48 trials. In the sixth block, the participants received 24 trials during which they practiced the reversed combination of targets and attributes. Finally, the seventh block was the reversed combination test block that consisted of 48 trials.

Table 1

Overview of the Implicit Association Test (IAT) Procedure

\begin{tabular}{cclll}
\hline Block & Trials & \multicolumn{1}{c}{ Function } & \multicolumn{1}{c}{ Left Key } & \multicolumn{1}{c}{ Right Key } \\
\hline 1 & 24 & Target practice & alcohol & soft drinks \\
2 & 24 & Attribute practice & positive & negative \\
3 & 24 & Combination practice & alcohol+positive & soft drinks + negative \\
4 & 48 & Combination test & alcohol+positive & soft drinks + negative \\
5 & 48 & Reversed target practice & soft drinks & alcohol \\
6 & 24 & Reversed combination practice & soft drinks + negative & alcohol+positive \\
7 & 48 & Reversed combination test & soft drinks+negative & alcohol+positive
\end{tabular}

Note-Blocks are shown for the bipolar IAT. In the unipolar IAT, the procedure was the same, except that the attribute categories were replaced with arousal versus neutral words. The assignment of the target and attribute categories to the left and right response keys was counterbalanced across participants. 
The assignment of the alcohol category and the soft drink category to the left and right response keys was counterbalanced across participants and was the same for the bipolar and unipolar IAT. Furthermore, the response assignment of the attribute categories was also counterbalanced, so that half the participants in each task performed the compatible combination task (i.e., alcohol + positive vs. soft drinks + negative for the bipolar IAT, and alcohol + arousal vs. soft drinks + neutral for the unipolar IAT) before the incompatible combination task (i.e., alcohol + negative vs. soft drinks + positive for the bipolar IAT, and alcohol + neutral vs. soft drinks + arousal for the unipolar IAT). The other half of the participants performed the incompatible combination task before the compatible combination task. During both IAT versions, the target and attribute stimuli were presented in the middle of the computer screen. During the task, the labels of the categories assigned to the left and right response keys were presented in the corresponding upper corners of the computer screen. The stimuli remained on the screen until a response was given. The intertrial interval was $250 \mathrm{msec}$. Feedback was presented in red beneath the stimuli after an incorrect response ("wrong") and when responses were too fast ( $<300 \mathrm{msec}$; "too fast") or too slow $(>3,000 \mathrm{msec}$; "too slow").

Explicit alcohol-related expectancies and attitudes. In line with previous research (e.g., Houben \& Wiers, 2006, 2007a, 2007b; Wiers et al., 2002; see also Wiers, 2008), positive and negative explicit alcohol-related expectancies were measured with an expectancy questionnaire that asked participants to indicate on a 7-point Likert scale how much they agreed $(1=$ completely disagree, $7=$ completely agree) with the statement, "After drinking alcohol, I feel . . .." Positive alcohol-related expectancies were measured by completing this statement with the following words: active, funny, energetic, cheerful, excited, pleasant, talkative, and happy. Negative alcohol-related expectancies were measured by completing the statement with the following words: miserable, awful, listless, nauseous, unpleasant, sad, and uncomfortable. In line with previous research (e.g., Houben \& Wiers, 2006, 2007a, 2007b; Wiers et al., 2002), explicit attitudes toward alcohol were assessed with four semantic differentials. The participants indicated on a 7-point Likert scale how much they considered drinking alcohol to be unpleasantpleasant, boring-fun, good-bad, and stupid-smart.

Alcohol use and alcohol-related problems. Alcohol use was measured through a self-report questionnaire (Wiers, Hoogeveen, Sergeant, \& Gunning, 1997) based on the timeline follow-back method (TLFB; Sobell \& Sobell, 1990). The participants were asked to indicate how many drinks of different types of alcoholic beverages they consumed during each day of the past week, and for each day of the week, how many drinks they typically consumed on this day of the week. Alcohol-related problems were assessed with the AUDIT. The AUDIT consisted of 10 questions in a multiple-choice format. The first three questions were related to alcohol use, the other seven to alcohol-related problems.

\section{Procedure}

The participants completed the bipolar and the unipolar IAT versions and the questionnaires related to their expectancies, attitudes, and drinking behavior once on their home computer via the Internet and once in the lab. The order of the home session and the lab session was counterbalanced, and the time interval between the two sessions was 2 weeks. During each session, the participants first performed the bipolar IAT and the unipolar IAT in balanced order. Next, the participants filled out the attitude questionnaire, the expectancy questionnaire, the TLFB, and the AUDIT, in that order. The participants, on average, needed about 20 min to perform the two IATs and about $30 \mathrm{~min}$ to fill out the questionnaires. Hence, each session had a duration of approximately $1 \mathrm{~h}$, and the total duration of the experiment (i.e., participation in both sessions) was approximately $2 \mathrm{~h}$.

During the home session, the participants accessed the Web site www.impliciet.eu, where they could log in using their user name and password. Next, they were directed to a page containing information about the experiment and a consent form. After giving consent, they received the IAT versions and the questionnaires. The online IAT versions were programmed in Macromedia Flash 8.0 Professional (2005). The online questionnaires were developed using Microsoft Frontpage (2002).

During the lab session, half the participants performed the IAT versions and completed the questionnaires via the Internet by $\log$ ging in on the Web site www.impliciet.eu. For these participants, the procedure for the lab session was the same as the procedure for the home session, with the difference that they now completed all the tasks and questionnaires in a standard, controlled lab setting. The other half of the participants received lab-based IAT versions that were programmed in Inquisit 2.0.610040.0 (2006). After completing the IAT versions, they were instructed to $\log$ in at the Web site, where they received the questionnaires in the online format. Following completion of the two sessions, the participants were thanked for their cooperation, received a gift certificate, and were provided with debriefing information

\section{RESULTS}

\section{IAT Effects}

IAT effects were calculated with the conventional log measure (Greenwald et al., 1998). First, trials with response latencies below $300 \mathrm{msec}$ or above $3,000 \mathrm{msec}$ were recoded to $300 \mathrm{msec}$ and $3,000 \mathrm{msec}$, respectively. For both the bipolar IAT and the unipolar IAT, mean response latencies were calculated for the compatible combination task and the incompatible combination task. Next, the conventional measure was calculated as the difference between these two log-transformed means, so that higher IAT scores indicate faster performance for the compatible response assignment (i.e., alcohol + pleasantlactive vs. soft drinks + unpleasant/neutral) than for the incompatible response assignment (i.e., alcohol + unpleasant/ neutral vs. soft drinks + pleasant/active). Preparatory analyses revealed no influential outliers on IAT data.

First, we examined the influence of the software (i.e., Inquisit or Internet) on the results with the IAT in the laboratory setting. The results demonstrated that the participants did not commit more errors when they performed the bipolar IAT via Inquisit in the laboratory $(6.21 \%$ errors $)$ or online in the laboratory $(6.53 \%$ errors) $[t(113)=0.35$, $p=.73, d=0.05]$. Similarly, there was no difference in the percentage of errors made with the Inquisit-based unipolar IAT in the laboratory (5.41\% errors) and the online unipolar IAT performed in the laboratory $(6.49 \%$ errors $)$ $[t(113)=1.13, p=.26, d=0.21]$. Next, we examined whether IAT effects measured in the laboratory via the Internet differed from IAT effects measured using Inquisit in the lab context. The results showed that this was not the case for the bipolar IAT $[t(113)=0.46, p=.65, d=0.09]$ or for the unipolar IAT $[t(113)=1.85, p=.07, d=0.35]$. For the unipolar IAT, however, there was a trend indicating that IAT effects measured with Inquisit were smaller in effect size than IAT effects assessed via the Internet.

Second, it was examined whether IAT effects are influenced by the setting (i.e., at home or in the lab). The results demonstrated that the participants did not make more errors with the bipolar IAT (6.67\% errors) and the unipolar IAT (6.47\% errors) performed at home, as compared with the bipolar IAT (6.38\% errors) and the unipolar IAT $(5.99 \%$ errors) performed in the lab $[t(114)=0.87$, 
$p=.39, d=0.08$, and $t(114)=1.60, p=.11, d=0.11$, respectively]. Furthermore, the results showed no difference between effects in the bipolar IAT when the task was performed at home or in the lab $[t(114)=0.26, p=.79$, $d=0.03]$. Similarly, there was no significant difference between unipolar IAT effects measured at home and unipolar IAT effects measured in the lab $[t(114)=-0.69$, $p=.52, d=0.08]$. Hence, IAT effects did not differ as a function of whether the participants performed the task at home or in the lab. Correlations between IAT effects from the home session and IAT effects measured during the lab session are shown in Table 2.

The effect with the bipolar IAT, averaged across the home session and the lab session, was significant $[t(114)=-19.79, p<.001, d=1.85]$, indicating that there was a reaction time difference between classification performance in the two combination tasks. Specifically, the participants were faster when alcohol-related stimuli and negative stimuli had to be classified with the same response than when alcohol-related stimuli and positive stimuli had to be classified with the same response. The effect with the unipolar IAT, also averaged across the home and lab sessions, was also significant $[t(114)=$ $6.50, p<.001, d=0.61]$, indicating that the participants were faster when alcohol-related stimuli and arousal stimuli had to be classified with the same response than when alcohol-related stimuli and neutral stimuli had to be classified with the same response.

\section{Relationship With Explicit Measures}

For both the home session and the lab session, mean expectancy scores were calculated separately for the positive and negative expectancy items. Both positive $(M=$ $4.85, S D=1.10)$ and negative $(M=2.71, S D=1.17)$ expectancy scores obtained from the home session did not differ significantly from the positive $(M=4.83, S D=$ $1.03)$ and negative $(M=2.74, S D=1.14)$ expectancy scores obtained during the lab session $[t(86)=0.25, p=$ $.81, d=0.02$, and $t(86)=-0.37, p=.71, d=0.03$, respectively]. Therefore, positive and negative expectancy scores were averaged across the home session and the lab session. ${ }^{2}$ Similarly, mean attitude scores calculated separately for the home session $(M=4.32, S D=1.11)$ and the lab session $(M=4.31, S D=1.02)$ also did not differ significantly $[t(86)=0.21, p=.84, d=0.01]$, and were therefore averaged (see note 2 ).

Correlations of explicit alcohol-related expectancies and attitudes with IAT effects are shown in Table 2. Only the IAT versions that were performed at home showed significant correlations with explicit measures, whereas none of the correlations between IAT versions performed in the lab and explicit measures reached significance. More specifically, for the participants who performed the IAT versions via the Internet in the lab as well as at home, the correlation between the unipolar IAT performed at home and positive expectancies was significant, and the correlation between the unipolar IAT and explicit attitudes toward alcohol was borderline significant. The bipolar IAT performed at home and the lab-based IAT versions, in contrast, did not show a relationship with explicit alcohol-related cognitions. For participants who performed the IAT versions via the Internet at home and via Inquisit in the lab, the results showed that the bipolar IAT performed at home was significantly correlated with explicit positive expectancies and explicit attitudes toward alcohol. Correlations between the bipolar IAT performed in the lab and explicit measures, however, were only borderline significant. Furthermore, the unipolar IAT was also significantly correlated with explicit attitudes when it was performed at home, but not when it was performed in the lab. Thus, overall, the IAT versions performed at home showed significant correlations with explicit measures, whereas the IAT versions performed in the lab did not. However, these correlations did not differ significantly from each other $(p s>.05)$ in either of the groups (i.e., lab Internet or lab Inquisit). Furthermore, there was an effect of software in such a way that the Inquisit-based lab version of bipolar IAT was more strongly correlated with both explicit positive expectancies $(z=-2.27, p=$ $.01)$ and explicit attitudes $(z=-2.17, p=.02)$ than was the Internet-based lab version of the bipolar IAT. With respect to the unipolar IAT, the difference in correlations

Table 2

Correlations of Bipolar and Unipolar Implicit Association Test (IAT) Versions Performed at Home or in the Lab With Explicit Cognitions, Shown Separately for Participants Who Performed the Lab IAT via Inquisit and Participants Who Performed the Lab IAT via the Internet

\begin{tabular}{|c|c|c|c|c|c|c|c|c|}
\hline & \multicolumn{4}{|c|}{ Home Versus Lab-Internet } & \multicolumn{4}{|c|}{ Home Versus Lab-Inquisit } \\
\hline & \multicolumn{2}{|c|}{ Home } & \multicolumn{2}{|c|}{$\mathrm{Lab}$} & \multicolumn{2}{|c|}{ Home } & \multicolumn{2}{|c|}{$\mathrm{Lab}$} \\
\hline & $\begin{array}{l}\text { Bipolar } \\
\text { IAT }\end{array}$ & $\begin{array}{c}\text { Unipolar } \\
\text { IAT }\end{array}$ & $\begin{array}{l}\text { Bipolar } \\
\text { IAT }\end{array}$ & $\begin{array}{c}\text { Unipolar } \\
\text { IAT }\end{array}$ & $\begin{array}{l}\text { Bipolar } \\
\text { IAT }\end{array}$ & $\begin{array}{c}\text { Unipolar } \\
\text { IAT }\end{array}$ & $\begin{array}{c}\text { Bipolar } \\
\text { IAT }\end{array}$ & $\begin{array}{c}\text { Unipolar } \\
\text { IAT }\end{array}$ \\
\hline \multicolumn{9}{|l|}{ Home } \\
\hline Bipolar IAT & - & & & & - & & & \\
\hline Unipolar IAT & .19 & - & & & .20 & - & & \\
\hline \multicolumn{9}{|l|}{$\mathrm{Lab}$} \\
\hline Bipolar IAT & $.50^{* * *}$ & .02 & - & & .23 & $.28^{*}$ & - & \\
\hline Unipolar IAT & $.29^{*}$ & $.23^{\dagger}$ & .13 & - & .08 & $.49^{* * *}$ & .12 & - \\
\hline Positive expectancies & .15 & $.32^{*}$ & -.15 & .14 & $.35^{*}$ & .07 & $.27^{\dagger}$ & .10 \\
\hline Negative expectancies & -.01 & -.04 & .01 & -.04 & -.22 & -.14 & .01 & -.16 \\
\hline Attitudes & .03 & $.22^{\dagger}$ & -.16 & -.01 & $.29^{*}$ & $.33^{*}$ & $.25^{\dagger}$ & .09 \\
\hline
\end{tabular}

Note-In the home versus lab via the Internet, $n=62$, and in the home versus lab via Inquisit, $n=53$. $t p \leq .10$ (twotailed). ${ }^{*} p<.05$ (two-tailed). ${ }^{* * *} p<.001$ (two-tailed). 
with explicit cognitions did not differ as a function of lab software $(p s>.05)$.

\section{Predicting Drinking Behavior}

For both the home session and the lab session, alcohol consumption during the past week was estimated from the TLFB. Because reported alcohol use in the week previous to the home session did not differ significantly from reported alcohol use in the week previous to the lab session $[t(68)=-1.10, p=.28, d=0.08]$, these two estimates were averaged (see note 2 ). Similarly, AUDIT scores measured at home and AUDIT scores obtained during the lab session did not differ significantly $[t(86)=-0.53, p=$ $.60, d=0.02]$. Therefore, an estimate of alcohol-related problems was calculated as the average of these two AUDIT scores (see note 2). Before the average alcohol use estimate and the average AUDIT score were entered in the correlational analyses and the regression analyses, these dependent variables were log-transformed. Furthermore, 26 participants with an average weekly alcohol consumption of 0 drinks were excluded from the following analyses in order to obtain a normal distribution for both dependent variables. This resulted in a sample of 89 participants for the analyses. In this sample, alcohol use and alcohol-related problems were significantly correlated $(r=.80, p<.001)$, indicating that the participants with increased levels of alcohol consumption also experienced more alcohol-related problems.

We examined the correlations of the IAT versions and explicit alcohol-related cognitions with alcohol use and alcohol-related problems. These correlational analyses were performed separately for the two lab conditions. The results are shown in Table 3. First, we examined the influence of setting on the relationship between the IAT versions and drinking behavior. When the participants performed the

Table 3

Correlations of Implicit Association Test (IAT) Versions and Explicit Measures With Drinking Behavior

\begin{tabular}{|c|c|c|}
\hline & Alcohol Use & Alcohol Problems \\
\hline \multicolumn{3}{|c|}{ Home Versus Lab-Internet } \\
\hline \multicolumn{3}{|l|}{ Home } \\
\hline Bipolar IAT & $.25^{\dagger}$ & $.32^{*}$ \\
\hline Unipolar IAT & $.36^{* *}$ & $.41^{* *}$ \\
\hline \multicolumn{3}{|l|}{$\mathrm{Lab}$} \\
\hline Bipolar IAT & -.06 & -.12 \\
\hline Unipolar IAT & .22 & .09 \\
\hline Positive expectancies & $.43^{* *}$ & $.39^{* *}$ \\
\hline Negative expectancies & -.22 & -.08 \\
\hline Attitudes & $.59^{* * *}$ & $.45^{* *}$ \\
\hline \multicolumn{3}{|c|}{ Home Versus Lab-Inquisit } \\
\hline \multicolumn{3}{|l|}{ Home } \\
\hline Bipolar IAT & .01 & .25 \\
\hline Unipolar IAT & $.32^{\dagger}$ & .24 \\
\hline \multicolumn{3}{|l|}{$\mathrm{Lab}$} \\
\hline Bipolar IAT & $.34^{*}$ & $.37^{*}$ \\
\hline Unipolar IAT & $.32^{*}$ & .18 \\
\hline Positive expectancies & $.34^{*}$ & $.61^{* * *}$ \\
\hline Negative expectancies & -.25 & -.20 \\
\hline Attitudes & $.56^{* * *}$ & $.68^{* * *}$ \\
\hline
\end{tabular}

Note-In the home versus lab via the Internet, $n=51$, and in the home versus lab via Inquisit, $n=38 . \quad{ }^{\dagger} p \leq .10$ (two-tailed). ${ }^{*} p<.05$ (twotailed). ${ }^{* *} p<.01$ (two-tailed). ${ }^{* * *} p<.001$ (two-tailed).
IAT versions via the Internet during both the home session and the lab session, both the bipolar IAT and the unipolar IAT that were performed at home showed a relationship with drinking behavior, but not the IAT versions that were performed in the lab. Specifically, the home version of the bipolar IAT showed a correlation with alcohol use that was borderline significant, as well as a significant correlation with alcohol-related problems, indicating that stronger positive implicit associations with alcohol are related to increased alcohol use and alcohol-related problems. The bipolar IAT that was performed in the lab, in contrast, was unrelated to both alcohol use and alcohol-related problems. Moreover, the correlations of the bipolar IAT with alcohol use and alcohol-related problems were significantly different between the home setting and the lab setting $(z=-2.18$, $p=.03$, and $z=-3.12, p=.002$, respectively). Furthermore, the home version of the unipolar IAT was correlated significantly with both alcohol use and alcohol-related problems, showing that stronger implicit associations between alcohol and arousal were associated with increased levels of alcohol consumption and more alcohol-related problems. The unipolar IAT that was performed in the lab setting, however, did not show significant correlations with drinking behavior. This difference in correlations with drinking behavior between the two settings was borderline significant for the correlations with alcohol-related problems $(z=-1.90, p=.06)$ but not with respect to alcohol use $(z=-.84, p=.40)$. Finally, both positive expectancies and attitudes toward alcohol were positively correlated with alcohol use and alcohol-related problems.

Similarly, we also examined the influence of setting on correlations between the IAT versions and drinking behavior for the group of participants who performed the IAT versions at home via the Internet and in the lab via Inquisit. In this group, the home version of the bipolar IAT was not significantly correlated with alcohol-related problems, whereas the correlation between the bipolar IAT performed at home and alcohol use approached significance. The lab version of the bipolar IAT, in contrast, correlated significantly with both alcohol use and related problems. The unipolar IAT was significantly correlated with alcohol use when the task was performed in the lab, but not when the task was performed at home. However, none of the correlations between the IAT versions performed at home and drinking behavior differed significantly from the correlations between the IAT versions performed in the lab and drinking measures ( $p$ s $>.05$ ). Finally, positive expectancies and attitudes toward alcohol were also significantly correlated with alcohol use and alcohol-related problems in this group.

Furthermore, we also examined the influence of software on the strength of the correlations between the labbased IAT versions and drinking behavior. The Inquisitbased lab version of the bipolar IAT showed significantly higher correlations with alcohol use and alcohol-related problems than did the Internet-based lab version of the bipolar IAT $(z=-1.86, p=.03$, and $z=-2.29, p=.01$, respectively). For the lab-based unipolar IAT, however, correlations with drinking behavior did not vary significantly as a function of software $(p \mathrm{~s}>.05)$. 
Table 4

Summary of Hierarchical Regression Analysis Predicting Alcohol Use

\begin{tabular}{clccccc}
\hline Step & \multicolumn{1}{c}{ Variable } & $B$ & $S E(B)$ & \multicolumn{1}{c}{$\beta$} & $S E(\beta)$ & \multicolumn{1}{c}{$t$} \\
\hline 1 & Positive alcohol expectancies & 0.11 & 0.08 & .15 & .10 & 1.42 \\
& Negative alcohol expectancies & 0.04 & 0.08 & .05 & .10 & 0.48 \\
& Attitude toward alcohol & 0.45 & 0.10 & .52 & .11 & $4.68^{* * *}$ \\
& Positive alcohol expectancies & 0.11 & 0.08 & .14 & .10 & 1.35 \\
& Negative alcohol expectancies & 0.02 & 0.08 & .03 & .10 & 0.28 \\
& Attitude toward alcohol & 0.40 & 0.10 & .47 & .11 & $4.17^{* * *}$ \\
& Bipolar IAT-home & -0.03 & 0.45 & -.01 & .09 & -0.06 \\
& Unipolar IAT-home & 1.11 & 0.53 & .19 & .09 & $2.11^{*}$ \\
2 & Positive alcohol expectancies & 0.08 & 0.08 & .10 & .10 & 1.00 \\
& Negative alcohol expectancies & 0.07 & 0.08 & .08 & .10 & 0.85 \\
& Attitude toward alcohol & 0.47 & 0.09 & .55 & .11 & $5.10^{* * *}$ \\
& Bipolar IAT-lab & 0.52 & 0.41 & .11 & .09 & 1.27 \\
& Unipolar IAT-lab & 1.24 & 0.48 & .23 & .09 & $2.60^{*}$ \\
\hline
\end{tabular}

Note- $F(3,85)=14.45, p<.001, R^{2}=.34$, for Step 1 . When the home implicit association test (IAT) versions were entered as predictors: $F_{\text {change }}(2,83)=2.22, p=.12, R_{\text {change }}^{2}=.03$, for Step 2; final model, $R^{2}=.37, R_{\text {adjusted }}^{2}=.33, F(5,83)=9.81, p<.001$. When the lab IAT versions were entered as predictors: $F_{\text {change }}(2,83)=4.44, p=.02, R_{\text {change }}^{2}=.06$, for Step 2; final model, $R^{2}=.40, R_{\text {adjusted }}^{2}=.37, F(5,83)=11.12, p<.001 . \quad{ }^{*} p<.05 . \quad{ }^{* * *} p<.001$.

The predictive validity of the explicit measures and the incremental validity of the IAT versions were tested using hierarchical regression analysis. To test incremental validity, explicit alcohol-related expectancies and attitudes toward alcohol were always entered in Step 1, and the IAT versions were entered in Step 2. Regression analyses were performed separately for the prediction of alcohol use and alcohol-related problems. Moreover, these regression analyses were performed twice, once with the home IAT versions as predictors and once with the lab IAT versions as predictors. The hierarchical regression analysis predicting alcohol use is shown in Table 4. In Step 1 of the regression analysis, attitudes toward alcohol significantly predicted alcohol use. When the home versions of the bipolar IAT and the unipolar IAT were entered in Step 2, the results showed that only the uni- polar IAT significantly predicted alcohol use above the variance explained by explicit attitudes toward alcohol. ${ }^{3}$ Similarly, when the lab versions of the bipolar and unipolar IAT were entered in Step 2, the unipolar IAT, but not the bipolar IAT, significantly predicted alcohol use above the variance explained by the explicit measures (see note 3 ). With respect to the prediction of alcohol use by the IAT versions that were performed in the lab, it was also examined whether there was a difference between the predictive validity of the Internet-based lab IAT versions and the Inquisit-based lab IAT versions. However, neither lab version (i.e., Internet or Inquisit) $(\beta=-.10$, $p=.56)$ nor any of the interactions between the lab version and the lab-based bipolar and unipolar IAT ( $\beta=.13$, $p=.69$, and $\beta=.35, p=.18$, respectively) significantly predicted alcohol use.

Table 5

Summary of Hierarchical Regression Analysis Predicting Alcohol-Related Problems

\begin{tabular}{clccccc}
\hline Step & \multicolumn{1}{c}{ Variable } & $B$ & $S E(B)$ & $\beta$ & $S E(\beta)$ & $t$ \\
\hline 1 & Positive alcohol expectancies & 0.13 & 0.04 & .31 & .10 & $3.09^{* *}$ \\
& Negative alcohol expectancies & 0.08 & 0.04 & .18 & .10 & $1.85^{\dagger}$ \\
& Attitude toward alcohol & 0.22 & 0.05 & .47 & .11 & $4.39^{* * *}$ \\
2 & Positive alcohol expectancies & 0.11 & 0.04 & .26 & .10 & $2.66^{* *}$ \\
& Negative alcohol expectancies & 0.07 & 0.04 & .17 & .10 & $1.80^{\dagger}$ \\
& Attitude toward alcohol & 0.20 & 0.05 & .42 & .11 & $3.97^{* * *}$ \\
& Bipolar IAT-home & 0.41 & 0.23 & .15 & .09 & 1.76 \\
& Unipolar IAT-home & 0.53 & 0.27 & .17 & .09 & $1.92^{\dagger}$ \\
& Positive alcohol expectancies & 0.12 & 0.04 & .29 & .10 & $2.87^{* *}$ \\
& Negative alcohol expectancies & 0.08 & 0.04 & .19 & .10 & $1.93^{*}$ \\
& Attitude toward alcohol & 0.23 & 0.05 & .48 & .11 & $4.46^{* * *}$ \\
& Bipolar IAT-lab & 0.24 & 0.23 & .09 & .09 & 1.07 \\
& Unipolar IAT-lab & 0.23 & 0.26 & .08 & .09 & 0.89 \\
\hline
\end{tabular}

Note-F(3,85) $=17.18, p<.001, R^{2}=.38$, for Step 1 . When the home implicit association test (IAT) versions were entered as predictors: $F_{\text {change }}(2,83)=3.59, p=.03$, $R_{\text {change }}^{2}=.05$, for Step 2; final model, $R^{2}=.43, R_{\text {adjusted }}^{2}=.39, F(5,83)=12.73, p<$ .001 . When the lab IAT versions were entered as predictors: $F_{\text {change }}(2,83)=1.03, p=$ $.36, R_{\text {change }}^{2}=.02$, for Step 2; final model, $R^{2}=.39, R_{\text {adjusted }}^{2}=.36, F(5,83)=10.73$, $p<.001 . \quad{ }^{\dagger} \leq .10 .{ }^{* *} p<.01 .{ }^{* * *} p<.001$. 
The hierarchical regression analysis for the prediction of alcohol-related problems is shown in Table 5. At Step 1, both positive expectancies and attitudes toward alcohol significantly predicted alcohol-related problems, whereas negative expectancies predicted alcohol-related problems at borderline significance. The home versions of the bipolar and unipolar IAT entered at Step 2 significantly increased the variance explained: The unipolar IAT significantly predicted alcohol-related problems above the variance explained by explicit measures, whereas the bipolar IAT was borderline significant (see note 3 ). In contrast, when the lab IAT versions were entered at Step 2, none of the IAT versions significantly predicted alcohol-related problems (see note 3 ). Furthermore, there was no difference between the predictive validity of the Internet-based lab IAT versions and the Inquisit-based lab IAT versions [lab version (i.e., Internet or Inquisit), $\beta=$ $.01, p=.96]$. Also, none of the interactions between the lab version and the lab-based bipolar and unipolar IAT significantly predicted alcohol use $(\beta=.22, p=.49$, and $\beta=.33, p=.21$, respectively).

\section{DISCUSSION}

The goal of the present study was to verify the validity of online administration of two recently developed alcohol IATs. To this end, we examined the influence of both software (Flash or Inquisit) and setting (assessment at home or in the lab) on results with the bipolar and unipolar alcohol IAT and the relationship of these IAT effects to explicit measures and drinking behavior. The present findings demonstrate robust effects with the alcohol IAT versions, regardless of setting and software. Moreover, the relationship of the IAT versions with explicit measures and drinking behavior for IAT was similar irrespective of whether data were collected at home or in the lab with either Inquisit or Flash. There were even some indications that the IAT versions performed at home were more strongly related to explicit measures and drinking behavior than were the lab-based IAT versions. Thus, these findings demonstrate that IAT versions can be validly administered online via participants' home computers.

First, with respect to the assessment of implicit alcoholrelated associations, the results revealed that the software used to produce online versions of the IAT yielded results similar to those for software that is typically used in the laboratory. Specifically, there was no difference between IAT effects in the online Flash-based IAT versions and IAT effects in the Inquisit-based IAT versions, indicating that the online IAT versions were as sensitive to individual differences in implicit alcohol-related associations as the Inquisit-based IATs. The present findings also demonstrate that administering the IAT in the setting of participants' own homes or in a standard laboratory setting does not influence results with the IAT. Furthermore, it is interesting to note that the present findings with both the bipolar IAT and the unipolar IAT replicated previous findings that were obtained in controlled laboratory settings. Consistent with earlier studies, the findings with the bipolar IAT showed that the participants had negative implicit associations with alcohol (cf. Houben \& Wiers, 2007b; Wiers et al., 2002), whereas the findings with the unipolar IAT indicated that the participants held implicit associations between alcohol and positive arousal (cf. Houben \& Wiers, 2006).

Second, regarding the relationship of IAT effects with explicit measures, the results showed that implicit alcohol associations were significantly related to explicit alcoholrelated expectancies and attitudes only when they were assessed in the home setting, not when measured in the lab setting, regardless of which software was used in the lab. Even though this difference between the home setting and the lab setting was not significant, these results suggest that IAT effects obtained in the home setting are somewhat more strongly related to explicit cognitions than are IAT effects obtained in the lab. In addition, online IAT versions performed at home were more strongly related to drinking behavior than were online IAT versions performed in the lab, and these differences were also significant. Whereas the lab-based IAT versions were not significantly related to drinking behavior, the results with the online IAT versions performed at home indicated that stronger positive implicit associations (or weaker negative implicit alcohol associations) as well as stronger implicit alcohol associations with arousal were related to increased levels of alcohol consumption and increased alcohol-related problems (cf. Houben \& Wiers, 2006, 2007b). However, for the group of participants who performed the IAT via the Internet at home and via Inquisit in the lab, the IATs performed in the lab showed a significant relationship with drinking behavior, whereas the IAT versions performed at home did not.

This discrepancy in results between the participants who received the lab-based IAT versions via Inquisit and the participants who received the lab-based IAT versions via the Internet suggests that Inquisit may produce more valid results than does Flash. The results indeed confirmed that the lab-based bipolar IAT showed stronger correlations with explicit cognitions, alcohol use, and alcohol-related problems in the former group than in the latter group, although these differences were not significant with respect to the lab-based unipolar IAT. It is important to note, however, that the IAT versions that were performed at home showed weaker correlations with drinking behavior among the participants who performed the lab-based IAT versions in Inquisit than among the participants who performed the lab-based IAT versions via the Internet (although this difference was not significant: $p$ s > .05). Therefore, another possible explanation could be that this difference in the pattern of correlations between the two groups was due to order effects. It should be noted that there were more abstainers present in the group of participants who performed the lab-based IAT versions via Inquisit than in the group of participants who received the lab-based IAT versions via the Internet. When the abstainers were removed from the analyses involving the drinking measures, fewer participants were left in the first group, as compared with the latter. More important, after removing the abstainers, the order of the home session and the lab session was still evenly divided in the 
group of participants who performed the lab-based IAT versions via the Internet. However, about $75 \%$ of the participants who performed the lab-based IAT versions via Inquisit took part in the lab session before participating in the home session. Therefore, order effects may have given the lab-based IAT versions an advantage over the IAT versions performed at home in this group, since effects with the IAT typically decrease with repeated administrations (e.g., Nosek, Greenwald, \& Banaji, 2006).

Finally, with respect to the prediction of alcohol use, the results show the same pattern of results, regardless of whether the bipolar IAT and the unipolar IAT were delivered online via participants' home computers or administered in the laboratory. Furthermore, there was also no influence of software on predictive validity of lab-based IAT versions. Both the unipolar IAT performed at home and the unipolar IAT performed in the lab predicted alcohol use beyond the variance explained by explicit measures: Stronger implicit alcohol associations with arousal predict increased alcohol consumption beyond the variance in drinking behavior explained by explicit expectancies and attitudes (cf. Houben \& Wiers, 2006). The bipolar IAT versions performed at home and in the lab, in contrast, did not show incremental predictive validity. Regarding prediction of alcohol-related problems, the unipolar IAT showed incremental predictive validity only when it was performed at home, but not when it was performed in the lab, regardless of whether this lab version was Internet based or Inquisit based. The bipolar IAT again did not show incremental predictive validity, regardless of setting or software.

In sum, the overall pattern of findings demonstrates that the IAT can be just as validly administered over the Internet in participants' home environments as in a standard lab setting. Moreover, some results even suggest that the IAT is more strongly related with explicit measures and shows better predictive validity with respect to drinking behavior when IAT data are collected over the Internet via participants' home computers. This result may be due to participants' answering more honestly and in a less socially desirable fashion on self-report questionnaires when these are administered in the anonymity of their own homes rather than in a lab context where there is typically an experimenter present (e.g., Joinson, 1999). Since selfreport measures are typically more sensitive to response bias than is the IAT (e.g., De Houwer, 2006), eliminating or reducing this response bias by collecting self-reports in complete anonymity may, thus, increase the relationship between self-report measures and the IAT. Another related possibility is that explicit alcohol-related cognitions and implicit alcohol-related associations may be more accessible in the context of their own homes than in a standard lab context, which is typically devoid of alcohol-related cues (cf. Krank et al., 2005). Hence, for these reasons, online assessment of alcohol-related cognitions and drinking behavior in a home setting may be more valid than lab-based assessment.

This is the first time that implicit alcohol-related associations have been obtained and validated using online administration of the IAT. Together, the present findings indicate that the IAT can be validly used to measure implicit alcohol-related associations via the Internet, even though data are collected using software different from that routinely used in the laboratory and even though the testing environment is different across participants. The implication is that, using these online IAT versions, it can be far easier to reach larger numbers of participants - and also nonstudent populations, including clinical populations - which enables further examination of the relationship between implicit alcohol-related associations and drinking behavior. In this respect, however, it should be noted that the present sample used to validate the online administration of the alcohol IAT was drawn from a nonclinical population. Therefore, future research first needs to examine whether the present findings with respect to the validity of the online alcohol IAT also generalize to clinical populations. Moreover, it is important to note that, according to dual-process models, there are other implicit processes that originate in the impulsive system that may play a role in addiction. At least three different implicit cognitive processes can be distinguished: implicit associations, attentional bias, and action tendencies (for an overview, see Wiers et al., 2007; Wiers \& Stacy, 2006b). Importantly, the assessment of attentional bias via the Internet has recently also been validated with respect to anxiety (MacLeod, Soong, Rutherford, \& Campbell, 2007), and we are beginning to study the possibility of measuring automatic action tendencies toward alcohol via the Internet. Thus, together, these findings indicate that different automatic responses of the impulsive system can be measured via the Internet. Furthermore, the Internet also opens up new possibilities for the online administration of new interventions that are designed to specifically target implicit processes (for an overview, see Wiers et al., in press). Although a procedure for changing attentional bias over the Internet has recently been validated in the context of anxiety (MacLeod et al., 2007), future research needs to examine whether new interventions aimed at changing implicit associations and action tendencies can also be delivered effectively via the Internet.

In conclusion, the present study verifies that the IAT can be validly used to measure implicit associations with alcohol via the Internet, thereby making the task more readily accessible to populations other than students within their home environments. This not only enables investigating the role of implicit associations in drinking behavior in a wider range of participants, but also facilitates research on the effectiveness of interventions in changing implicit alcohol-related associations.

\section{AUTHOR NOTE}

This research was supported by a grant from the Technology Foundation STW. Our thanks go out to Charlie Bonnemayer and Michiel Vestjens for their help in the development of the tasks and questionnaires. We also thank the team of Project Implicit for their technical support with the setup of the online IAT versions. Finally, we thank Dilana Schaafsma for her assistance in testing the participants. Correspondence concerning this article should be addressed to K. Houben, Clinical Psychological Science, Maastricht University, P.O. Box 616, 6200 MD Maastricht, The Netherlands (e-mail: k.houben@psychology.unimaas.nl). 


\section{REFERENCES}

Ames, S. L., Grenard, J. L., Thush, C., Sussman, S., Wiers, R. W., \& STACY, A. W. (2007). Comparison of indirect assessments of marijuana-related associations among at-risk adolescents. Experimental \& Clinical Psychopharmacology, 15, 204-218.

De Houwer, J. (2006). What are implicit measures and why are we using them? In R. W. Wiers \& A. W. Stacy (Eds.), Handbook of implicit cognition and addiction (pp. 11-28). Thousand Oaks, CA: Sage.

De Houwer, J., Crombez, G., Koster, E. H. W., \& De Beul, N. (2004). Implicit alcohol-related cognitions in a clinical sample of heavy drinkers. Journal of Behavior Therapy \& Experimental Psychiatry, 35, 275-286.

Deutsch, R., \& Strack, F. (2006). Reflective and impulsive determinants of addictive behavior. In R. W. Wiers \& A. W. Stacy (Eds.), Handbook of implicit cognition and addiction (pp. 45-57). Thousand Oaks, CA: Sage.

Evans, J. S. B. T. (2003). In two minds: Dual-process accounts of reasoning. Trends in Cognitive Sciences, 7, 454-459.

Evans, J. S. B. T., \& Coventry, K. (2006). A dual process approach to behavioral addiction: The case of gambling. In R. W. Wiers \& A. W. Stacy (Eds.), Handbook of implicit cognition and addiction (pp. 29-43). Thousand Oaks, CA: Sage.

FrontPage 2002 [Computer SOFtware] (2002). Redmond, WA: Microsoft Corporation.

Greenwald, A. G., McGhee, D. E., \& Schwartz, J. L. K. (1998). Measuring individual differences in implicit cognition: The implicit association test. Journal of Personality \& Social Psychology, 74, 1464-1480.

Houben, K., \& Wiers, R. W. (2006). Assessing implicit alcohol associations with the Implicit Association Test: Fact or artifact? Addictive Behaviors, 31, 1346-1362.

Houben, K., \& Wiers, R. W. (2007a). Are drinkers implicitly positive about drinking alcohol? Personalizing the alcohol IAT to reduce negative extrapersonal contamination. Alcohol \& Alcoholism, 42, 301307.

Houben, K., \& Wiers, R. W. (2007b). Personalizing the alcohol IAT with individualized stimuli: Relationship with drinking behavior and drinking-related problems Addictive Behaviors, 32, 2852-2864.

Houben, K., Wiers, R. W., \& Roefs, A. (2006). Reaction time measures of substance-related associations. In R. W. Wiers \& A. W. Stacy (Eds.), Handbook of implicit cognition and addiction (pp. 91-104). Thousand Oaks, CA: Sage.

InQuisit 2.0.6IoO4.o [COMPUTER SOFTwARE] (2006). Seattle, WA: Millisecond Software LLC.

Jornson, A. N. (1999). Social desirability, anonymity, and Internet-based research questionnaires. Behavior Research Methods, 31, 433-438.

Krank, M., Wall, A., Stewart, S. H., Wiers, R. W., \& Goldman, M. S. (2005). Context effects on alcohol cognitions. Alcoholism: Clinical \& Experimental Research, 29, 196-206.

MacLeod, C., Soong, L. Y., Rutherford, E. M., \& CAmpbell, L. W. (2007). Internet-delivered assessment and manipulation of anxietylinked attentional bias: Validation of a free-access attentional probe software package. Behavior Research Methods, 39, 533-538.

Macromedia Flash 8.0 Professional [COMPUTER SOFtware] (2005). San Jose, CA: Adobe Systems.

Nosek, B. A., Greenwald, A. G., \& BanajI, M. R. (2006). The Implicit Association Test at age 7: A methodological and conceptual review. In J. A. Bargh (Ed.), Social psychology and the unconscious: The automaticity of higher mental processes (pp. 265-292). New York: Psychology Press.

Saunders, J. B., Aasland, O. G., Babor, T. F., De la Fuente, J. R., \& Grant, M. (1993). Development of the Alcohol Use Disorders Identification Test (AUDIT): WHO collaborative project on early detection of persons with harmful alcohol consumption. Addiction, 88, 791-804.

Sobell, L. C., \& Sobell, M. B. (1990). Self-report issues in alcohol abuse: State of the art and future directions. Behavioral Assessment, 12, 77-90.

Strack, F., \& Deutsch, R. (2004). Reflective and impulsive determinants of social behavior. Personality \& Social Psychology Review, 8, 220-247.

Thush, S., Wiers, R. W., Ames, S. L., Grenard, J. L., Sussman, S., \& StACY, A. W. (2007). Apples and oranges? Comparing indirect measures of alcohol-related cognition predicting alcohol use in at-risk adolescents. Psychology of Addictive Behaviors, 21, 587-591.

WIERS, R. W. (2008) Alcohol and drug expectancies as anticipated changes in affect: Negative reinforcement is not sedation. Substance Use \& Misuse, 43, 501-516.

Wiers, R. W., Bartholow, B. D., van den Wildenberg, E., Thush, C., Engels, R. C. M. E., Sher, K. J., ET AL. (2007). Automatic and controlled processes and the development of addictive behaviors in adolescents: A review and a model. Pharmacology Biochemistry \& Behavior, 86, 263-283.

Wiers, R. W., Cox, W. M., Field, M. Fadardi, J. S., Palfai, T. P., Schoenmakers, T., \& Stacy, A. W. (2006). The search for new ways to change implicit alcohol-related cognitions in heavy drinkers. Alcoholism: Clinical \& Experimental Research, 30, 320-331.

Wiers, R. W., Hoogeveen, K.-J., Sergeant, J. A., \& Gunning, W. B. (1997). High- and low-dose alcohol-related expectancies and the differential associations with drinking in male and female adolescents and young adults. Addiction, 92, 871-888.

Wiers, R. W., Schoenmakers, T., Houben, K., Thush, C., Fadardi, J. S., \& Cox, W. M. (in press). Can problematic alcohol use be trained away? New behavioural treatments aimed at changing and moderating implicit cognitive processes in alcohol abuse. In C. R. Martin (Ed.), Identification and treatment of alcohol dependency. Keswick, U.K.: M\&K Publishing.

Wiers, R. W., \& Stacy, A. W. (Eds.) (2006a). Handbook of implicit cognition and addiction. Thousand Oaks, CA: Sage.

Wiers, R. W., \& STACY, A. W. (2006b). Implicit cognition and addiction. Current Directions in Psychological Science, 15, 292-296.

Wiers, R. W., van Woerden, N., SMulders, F. T. Y., \& de Jong, P. J. (2002). Implicit and explicit alcohol-related cognitions in heavy and light drinkers. Journal of Abnormal Psychology, 111, 648-658.

\section{NOTES}

1. A standard alcoholic drink in Holland contains somewhat less alcohol than does a standard American alcoholic drink: 10 vs. $14 \mathrm{~g}$, respectively.

2. For 28 participants, responses to the expectancy and attitude questionnaires were missing for either the home session (13 participants) or the lab session (15 participants). These participants were excluded from the analyses that compared scores on the explicit measures between the home session and the lab session. Furthermore, since these participants had scores on the explicit measures for only one of the two sessions, these single scores were used to estimate explicit cognitions. The same 28 participants also had missing data on the AUDIT for either the home session or the lab session. For the participants who had only one AUDIT score, this score was used as an estimate of alcohol-related problems. Finally, 46 participants (including the 28 participants who produced missing data on the explicit measures and the AUDIT), reported alcohol use only during the home session (17 participants) or the lab session (29 participants). Consequently, for these participants, only data from this completed session were used as the estimate of average weekly alcohol use. When all analyses were performed without the 46 participants who produced missing data on one of the dependent measures, the pattern of results remained the same.

3. The results were almost identical when the bipolar IAT and the unipolar IAT were entered separately in the regression analyses.

(Manuscript received February 1, 2008; revision accepted for publication May 22, 2008.) 